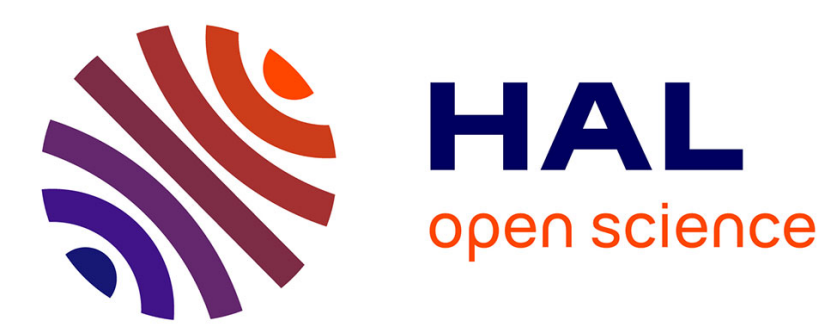

\title{
Hétérogénéité des réservoirs naturels carbonatés de pétrole et de gaz: exemple des réservoirs intersalifères du bassin de Pripiat (Biélorussie)
}

\author{
C. Kaki, E. Taranenko, A. Ryjkov
}

\section{- To cite this version:}

C. Kaki, E. Taranenko, A. Ryjkov. Hétérogénéité des réservoirs naturels carbonatés de pétrole et de gaz: exemple des réservoirs intersalifères du bassin de Pripiat (Biélorussie). Oil \& Gas Science and Technology - Revue d'IFP Energies nouvelles, 2000, 55 (5), pp.485-500. 10.2516/ogst:2000036 . hal-02054060

\author{
HAL Id: hal-02054060 \\ https://hal.science/hal-02054060
}

Submitted on 1 Mar 2019

HAL is a multi-disciplinary open access archive for the deposit and dissemination of scientific research documents, whether they are published or not. The documents may come from teaching and research institutions in France or abroad, or from public or private research centers.
L'archive ouverte pluridisciplinaire HAL, est destinée au dépôt et à la diffusion de documents scientifiques de niveau recherche, publiés ou non, émanant des établissements d'enseignement et de recherche français ou étrangers, des laboratoires publics ou privés. 


\title{
Hétérogénéité des réservoirs naturels carbonatés de pétrole et de gaz : exemple des réservoirs intersalifères du bassin
de Pripiat (Biélorussie)
}

\author{
C. Kaki', E. Taranenko ${ }^{2}$ et A. Ryjkov ${ }^{2}$ \\ 1 Département des sciences de la terre, FAST, Université nationale du Bénin, BP 526, Cotonou - Bénin \\ 2 Université russe de l'amitié des peuples, 3, rue Orjénikidzé, Moscou - Russie \\ e-mail : kaki@syfed.bj.refer.org
}

\begin{abstract}
Résumé - Le bassin de Pripiat, situé au sud-est de la Biélorussie, représente un grand graben qui a connu les cycles calédonien, hercynien et alpin dans l'histoire de la formation de sa couverture sédimentaire. À l'hercynien, on assiste à la formation d'un rift caractérisé par le dépôt de deux couches salifères. Ces dernières renferment un complexe sulfato-carbonaté du Dévonien supérieur dont la formation est liée à des variations de la profondeur des fonds du bassin induites par la tectonique et le volcanisme à l'est du bassin.

Il ressort de l'étude pétrographique que les réservoirs naturels du complexe intersalifère sont constitués de carbonates secondaires (calcaires, dolomies et variétés intermédiaires). Les transformations secondaires des calcaires et calcaires magnésiens s'intensifient de la base vers le sommet des anticlinaux avec l'augmentation des fissurations, et de l'ouest vers l'est du bassin sous l'influence croissante du volcanisme. Elles modifient la porosité et contribuent à la formation des roches magasins, dont la répartition irrégulière reflète la nature hétérogène des réservoirs naturels. Dans l'ensemble du complexe carbonaté intersalifère, il se dégage, sur la base de la densimétrie, sept types de roches avec une porosité ouverte limite de $2 \%$ entre les roches magasins et les fausses couvertures, et trois types de réservoirs naturels obtenus à partir des données géophysiques sur la porosité : tabulaire homogène à l'ouest, tabulaire hétérogène au centre et massif homogène à l'est de la zone d'étude.
\end{abstract}

Mots-clés : bassin de Pripiat, réservoir naturel, porosité ouverte (kpo), hétérogénéité, calcaire, dolomite, micrite, roche magasin, fausse couverture, complexe intersalifère, Claster Analyse.

Abstract - Heterogeneity of Oil and Gas Carbonate Natural Reservoirs: Exempt of Intersalt Reservoirs in Pripiat Basin (Byelorussia) - The Pripiat basin in the southeast of Byelorussia has known Caledonian, Hercynian and Alpian cycles in the geologic history of its sedimentary formation. At the Hercynian cycle, there is a rift characterized by two evaporitic formations, which contain a Devonian carbonate complex. This last was formed in salt active tectonic with basin bottom variations and volcanism at the east of the basin.

The petrographical analysis shows that the reservoir rocks are secondary carbonates (calcareous and dolomites) which are being transformed. These secondary processings intensify from the base to the fissured summit of the anticlines, and from western to eastern regions where the volcanic influence 
increases constantly. They modify the carbonate porosity and form the reservoir rocks. The irregular repartition of those makes them heterogeneous. The dynamic densimetry shows seven rock types with limited open porosity (2\%) between reservoir rocks and false coverings.

Using geophysical porosity data allows to stand out the horizontal and vertical repartition of reservoirs and false coverings. There correlation put in a prompt position three different natural reservoirs types: tabular homogeneous in the west, tabular heterogeneous in the center and massif homogeneous in the east of the studied region.

Keywords: Pripiat basin, natural reservoir, open porosity (kpo), heterogeneity, calcareous, dolomite, micrite, reservoir rock, false covering, intersalt complex, Claster Analysis.

\section{INTRODUCTION}

L'hétérogénéité macro- et microscopique des réservoirs naturels carbonatés du complexe intersalifère du bassin de Pripiat, qui se traduit par des changements structuraux de l'espace poreux et une répartition spatiale irrégulière des roches magasins, fausses couvertures et barrières de perméabilité, rend difficile la prospection et l'exploitation des gisements pétroliers qu'ils contiennent.

Dans le souci de maîtriser les particularités de ces réservoirs pour une meilleure productivité, diverses interprétations des diagraphies ont été effectuées avec l'appui des données de carottage. Ces travaux ont conduit à la subdivision lithologique du massif carbonaté en intervalles calcaires, dolomitiques et évaporitiques, puis à la détermination des porosités et teneurs en argile des couches (Anpilogov et Gossoudareva, 1974, 1975, 1987 ; Zaliaev et al., 1981), ainsi que d'une porosité limite de 4,8\% entre réservoirs et couvertures (Massiouk et Araktchev, 1976), sans toutefois qu'il soit tenu compte de l'évolution de la perméabilité avec la baisse de la pression des couches.

En effet, pour ces réservoirs carbonatés hétérogènes, au cours de l'exploitation des gisements et au fur et à mesure que baisse la pression des couches, les fausses couvertures se transforment en barrières de perméabilité, provoquant ainsi l'éclatement du gisement homogène en plusieurs petits gisements isolés dont l'exploitation doit se faire selon un schéma particulier. C'est pourquoi des données fiables sur la composition interne des réservoirs naturels, qui permettent de déterminer le type de gisement, l'orientation des travaux de prospection et le choix du système d'exploitation, paraissent d'importance capitale. L'implantation des puits d'exploitation doit être argumentée par une information pronostique sur la composition des réservoirs naturels.

C'est dans ce cadre que notre étude de l'hétérogénéité des réservoirs intersalifères dans la zone septentrionale du bassin de Pripiat apporte une contribution à la résolution du problème de la recherche pétrolière. Elle est basée sur l'interprétation du matériel géologique (échantillons de roches) et géophysique (diagraphies gamma et neutrongamma) de quatre champs : Davidovskaya, Dubrovskaya, Yuzno-Sosnovskaya et Yuzno-Alexandrovskaya, dans l'intervalle des couches intersalifères.

\section{CADRE GÉOLOGIQUE}

Le bassin de Pripiat, situé dans la partie sud-est de la Biélorussie, est une structure négative de $250 \times 150 \mathrm{~km}$, où les travaux de prospection pétrolière ont commencé activement dans les années 1940. Il occupe la partie sudouest de la plate-forme précambrienne est-européenne et fait partie du grand graben de Pripiat-Donetz qui occupe l'est de la Biélorussie et une partie de l'Ukraine (fig. la).

L'étude détaillée des caractéristiques lithologiques et stratigraphiques du bassin, réalisée par Gorelik (1968), Ayzberg et al. (1974) et Mahnatch (1984), donne des précisions sur la coupe géologique. On y distingue le socle cristallin et la couverture sédimentaire (fig. 1b, lc et 2).

Le socle est composé de granites, de granodiorites et de gneiss de l'Archéen et du Protérozoïque. La couverture sédimentaire comprend les formations du Protérozoïque supérieur, du Dévonien moyen et supérieur, du Carbonifère, du Permien puis des Secondaire et Tertiaire. Ces formations se subdivisent en trois grands complexes lithologiques :

- le complexe inférieur, composé de couches terrigènes rougeâtres du Protérozoïque supérieur (Rifain-Vendéen);

- le complexe moyen englobe les couches du Dévonien moyen et supérieur, du Carbonifère et du Permien. Il se subdivise en trois étages historico-génétiques : anté-rift, rift et post-rift (Ouriev et al., 1974 ; Moskvitch, 1990). L'étage inférieur est constitué de formations terrigènes et sulfato-carbonatées du Dévonien moyen et en partie supérieur. Ce sont des dolomies, des marnes, des calcaires argileux, des sables, des anhydrites et des argiles de l'Eifélien, du Givétien et de la base du Frasnien (horizons Lans, Sargaev, Sémilour, Rétchitsa, Voronèj et Evlanov) (fig. 2). L'étage moyen se compose de formations sulfatocarbonatées avec alternance de sel et de formations volcaniques du Dévonien supérieur. Ce sont les roches du complexe salifère inférieur dans la partie supérieure du Frasnien (horizon Liven), du complexe intersalifère au sommet du Frasnien (horizon Domanovitch) et à la base du Famennien (horizons Zadon, Eliets et Pétrikov), puis des complexes salifère supérieur (horizons Lébédian, Ores et en partie Strouchine) et sus-salifère (horizons Strouchine et Poles) dans la partie supérieure du Famennien (fig. 2). L'étage supérieur est formé de couches 


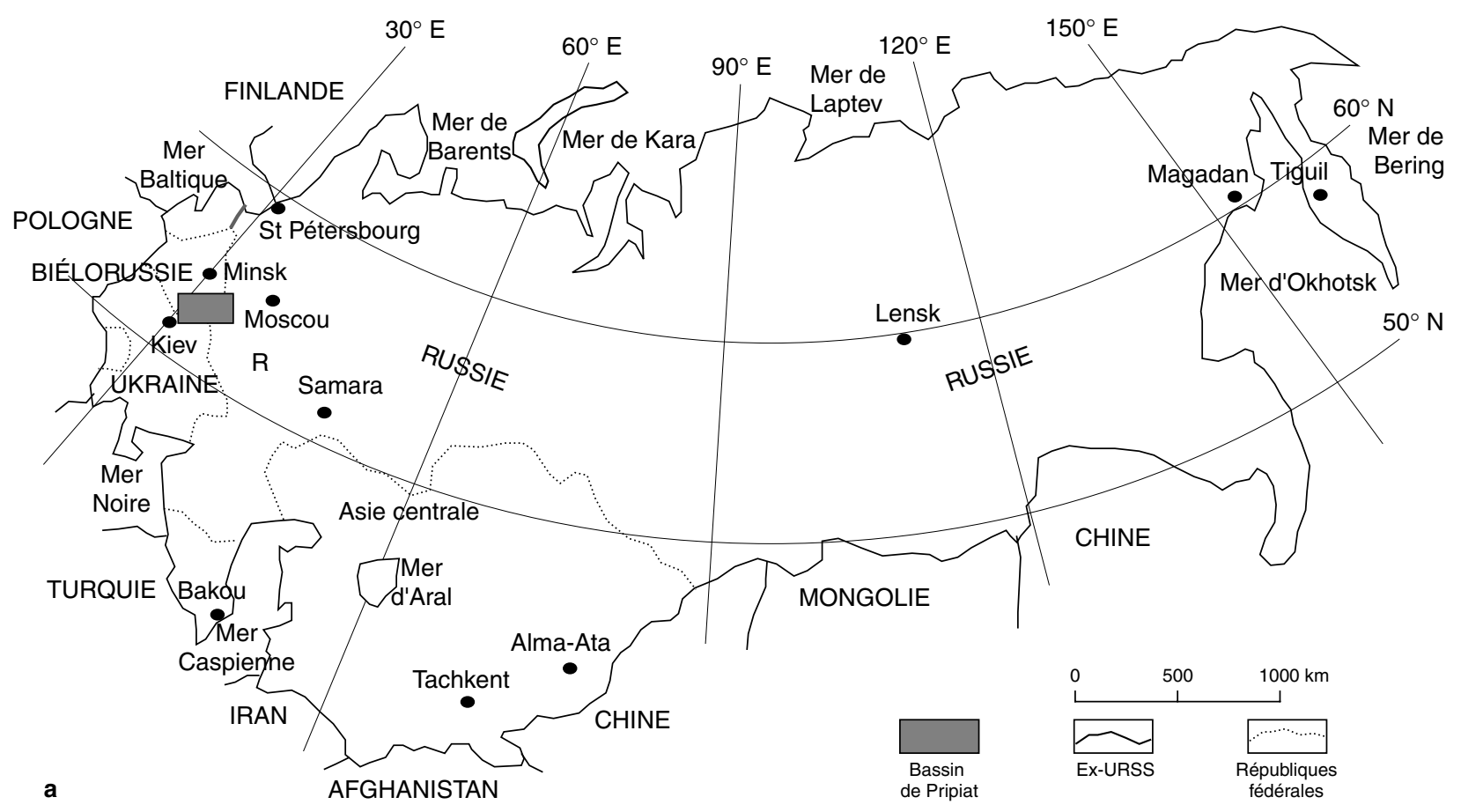

Figure 1

Schéma tectonique et profil géologique S-N du bassin de Pripiat (d'après Garetsky et al., 1984).

(a) Situation géographique du bassin de Pripiat en territoire de l'ex-URSS.

Tectonic sketch and geologic S-N cross-section of Pripiat basin (from Garetsky et al., 1984).

(a) Geographical location of Pripiat basin in ex-USSR.

terrigènes et argilo-carbonatées du Carbonifère et du Permien inférieur. Les formations de l'étage riftogénique occupent plus des deux tiers de l'épaisseur totale de la couverture sédimentaire du bassin (fig. 2);

- le complexe supérieur d'âge méso-cénozoïque est constitué de couches de faible épaisseur dont la formation correspond à la période de submersion du bassin durant le cycle alpin.

Le socle cristallin de l'Archéen et du Protérozoïque est cisaillé par plusieurs failles, parmi lesquelles on distingue des failles listriques d'orientation est-ouest, qui servent de limites au bassin, et des failles régionales est-ouest qui subdivisent le bassin en trois zones structurales différentes : méridionale, centrale et septentrionale (Pap, 1977).

L'histoire géologique du bassin de Pripiat révèle une liaison étroite entre la géodynamique et la pétrogenèse. On observe plusieurs étapes dans l'évolution de ce bassin : la base correspond au Protérozoïque tardif, sous forme de couches peu épaisses ayant résisté à l'érosion précédente. Au cycle calédonien, la région se présentait sous forme de zone de dénudation. L'activation de la région a commencé au Dévonien moyen (cycle hercynien), avec pour la première fois la formation des failles profondes de direction est-ouest, la disjonction des plaques et le dépôt des sédiments de plateforme dans le fossé. Au Dévonien tardif (Frasnien et Famennien), on assiste à la formation de deux couches évaporitiques (salifères) séparées par un faisceau de couches carbonatées et parfois volcaniques à l'est du bassin (Ouriev et al., 1974 ; Moskvitch, 1990). Ce fut le temps de la mise en place d'une gigantesque zone de rift allant de la mer Caspienne jusqu'en Pologne et passant par le Dombasse et le bassin de Pripiat.

L'étape du Famennien précoce (Zadon-Eliets-Pétrikov) est considérée comme la plus importante dans la formation du rift. Au Zadon, la sédimentation s'est déroulée dans des conditions diverses, allant de la côte au large. À certains endroits, on note des formations récifales correspondant aux compartiments relevés des failles régionales, et constituées de calcaires oncolitiques et stromatolitiques à porosité primaire et perméabilité élevées. À la fin de la diagenèse et au début de l'épigenèse, ces calcaires ont été fortement dolomitisés (Karsev et al., 1972 ; Sahibgareev et Moskvitch, 1975).

À l'Eliets, le volcanisme reprend à l'est du bassin, formant d'épaisses couches volcaniques. Au Pétrikov, l'approfondissement du bassin, qui avait commencé au Zadon- 


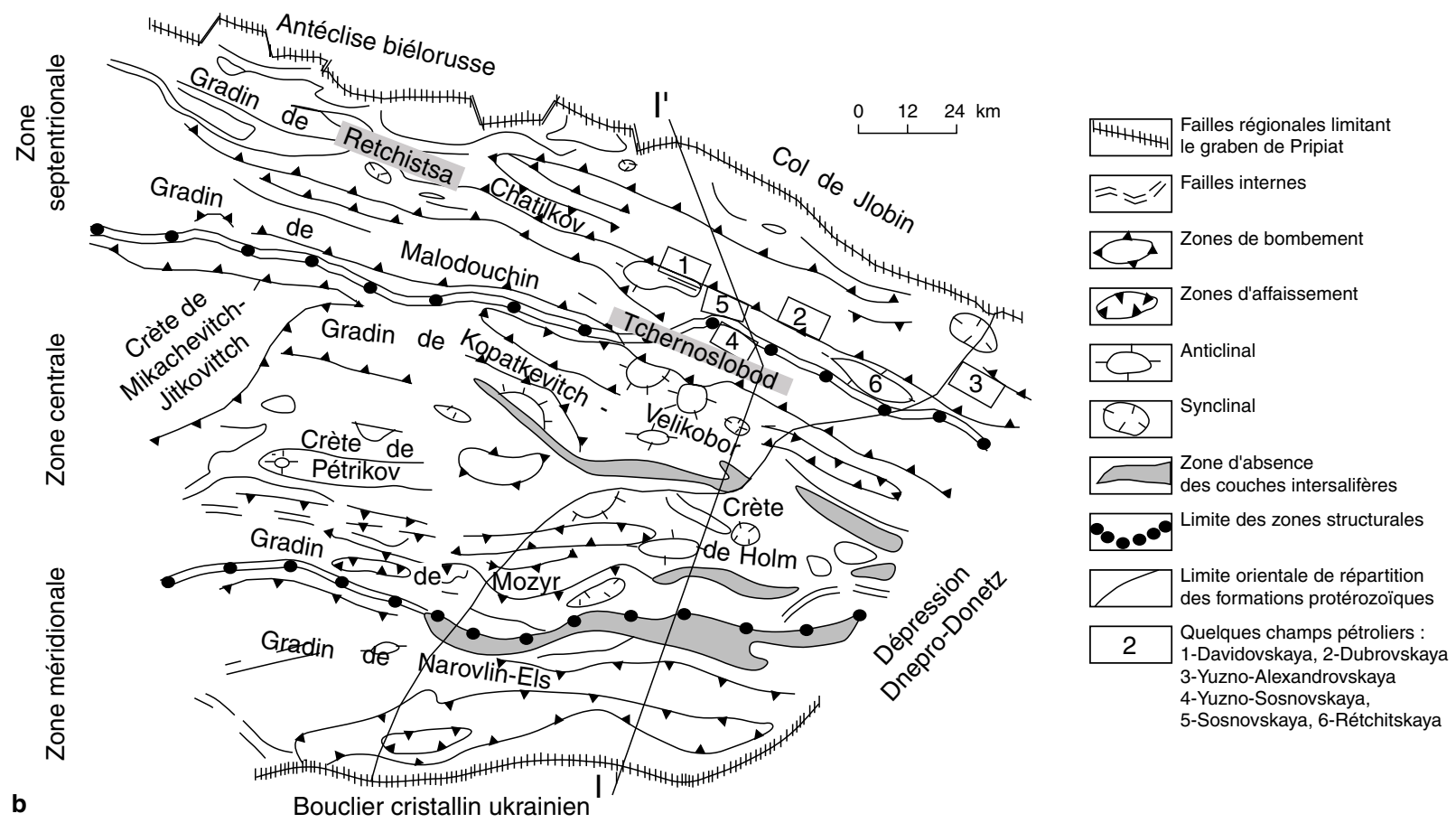

$S$

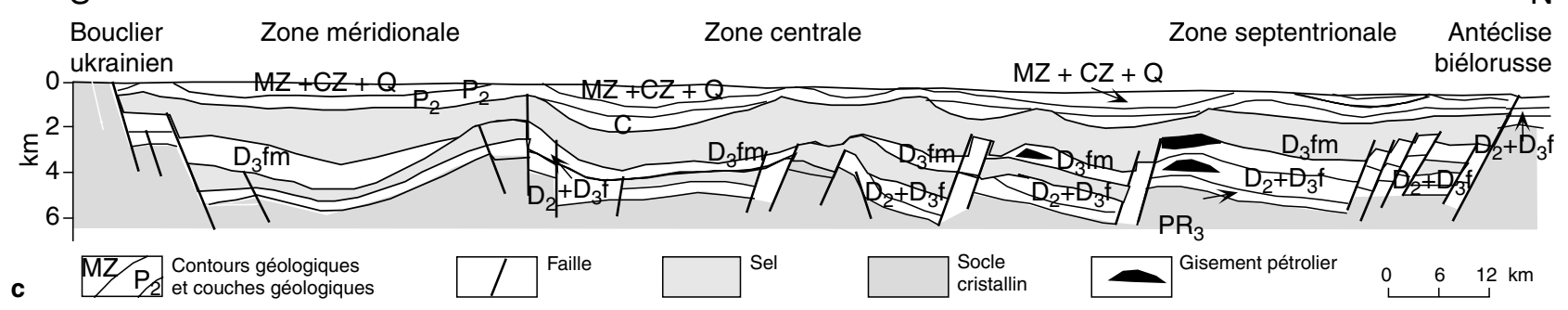

Figure 1

Schéma tectonique et profil géologique S-N du bassin de Pripiat (d'après Garetsky et al., 1984).

(b) Schéma tectonique.

(c) Profil géologique S-N suivant le trait I-I'.

Tectonic sketch and geologic S-N cross-section of Pripiat basin (from Garetsky et al., 1984).

(b) Tectonic sketch.

(c) Geologic cross-section (S-N) according to I-I'.

Eliets, s'est poursuivi et le bouclier ukrainien était à l'origine des sédiments terrigènes pour le paléobassin. À l'est, on note une diminution de l'activité volcanique. Le Famennien tardif est marqué par la formation périodique des couches calcaires et dolomitiques entre les formations évaporitiques. Plus tard, à la fin du Paléozoïque, la salinité du bassin se normalise, la riftogenèse tire sur sa fin et on assiste à un début de dépôts terrigènes de plate-forme, de faible épaisseur.
Au début du Mésozoïque, il se produit une stabilisation de l'écorce terrestre en Biélorussie et une longue période mésocénozoïque (cycle alpin) de sédimentation en mer peu profonde périodiquement asséchée.

Sur le plan pétrolier, le bassin présente un grand intérêt économique et fait partie de l'énorme bassin pétrolier DonetzPripiat. Les principaux complexes producteurs d'huile sont liés aux formations intersalifères et sous-salifères du 


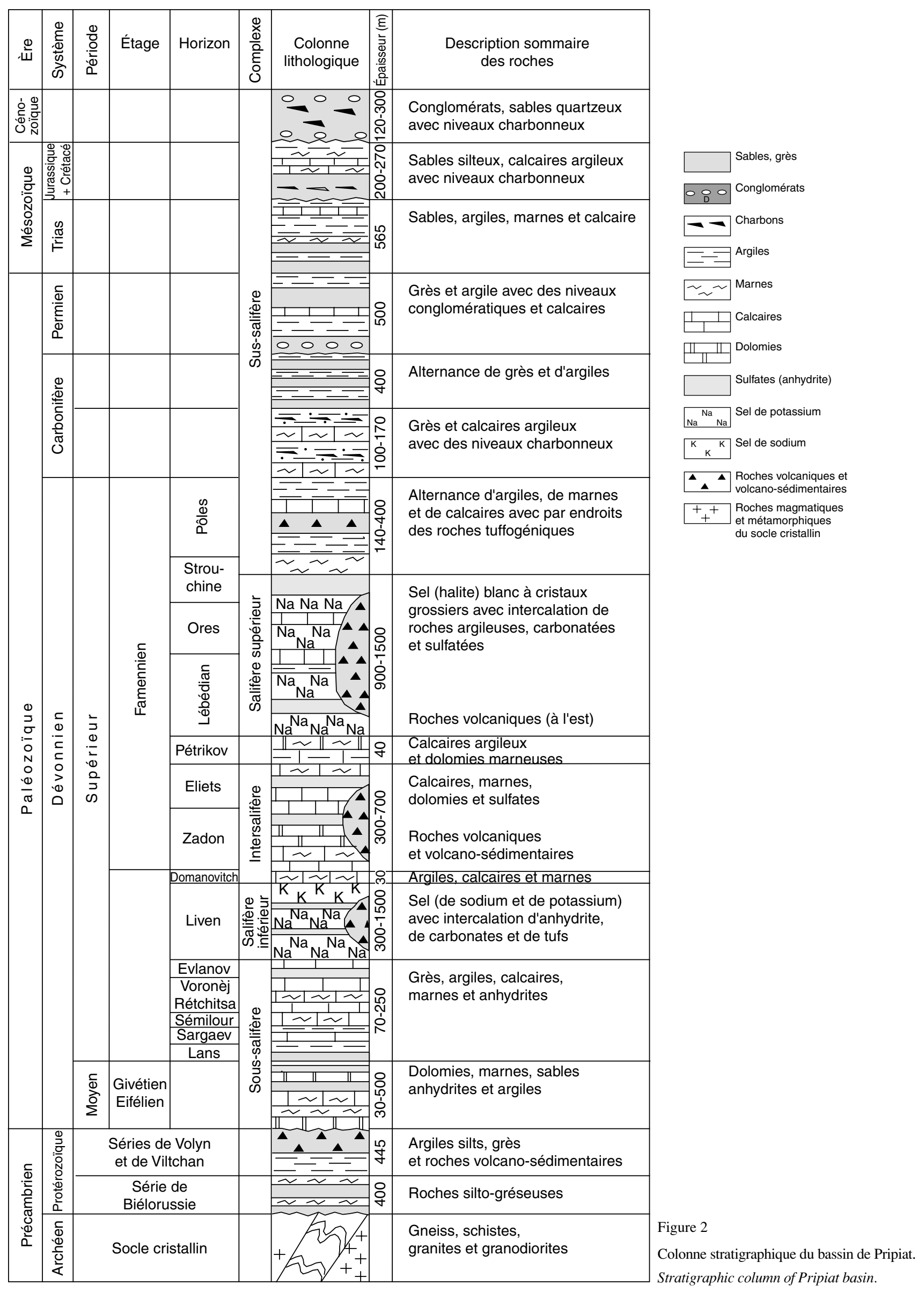


Dévonien supérieur. Des indices de pétrole se rencontrent le long de toute la coupe de la couverture sédimentaire. Les gisements de pétrole sont liés aux flancs des failles d'effondrement et au biseautage des couches carbonatées.

Les huiles sont paraffineuses légères et peu sulfureuses dans la zone structurale septentrionale et paraffineuses lourdes très sulfureuses dans les parties centrale et méridionale du bassin. Dans l'ensemble, les huiles intersalifères sont plus lourdes $\left(855 \mathrm{~kg} / \mathrm{m}^{3}\right)$ et beaucoup plus réparties au nord que les huiles sous-salifères $\left(835 \mathrm{~kg} / \mathrm{m}^{3}\right)$ réparties au sud. Les pressions de saturation sont de 5-6 MPa dans les gisements intersalifères et de 25-27 MPa dans les couches sous-salifères (Garetsky et al., 1984).

\section{CARACTÉRISTIQUES STRUCTURALES DES RÉSERVOIRS}

Les champs étudiés présentent des structures anticlinales limitées par des failles régionales et locales (fig. 3). Les champs Dubrovskaya et Davidovskaya sont situés sur les flancs relevés, tandis que les champs Yuzno-Sosnovskaya et Yuzno-Alexandrovskaya se trouvent sur le bloc affaissé sud des failles.
Les couches intersalifères du Frasnien supérieur et du Famennien inférieur qui font l'objet de notre étude se composent de bas en haut des horizons bien continus suivants (Anpilogov et Gossoudareva, 1974) :

- Domanovitch $\left(\mathrm{D}_{3} \mathrm{dm}\right)$, dans la partie supérieure du Frasnien et à la base du Famennien, d'une épaisseur de $20 \mathrm{~m}$, est formé de calcaires marneux. À l'est du bassin, il est constitué de roches effusives issues du volcanisme ayant affecté cette localité. Cet horizon repose partout sur du sel de l'horizon Liven $\left(D_{3} l v\right)$ du Frasnien ;

- Zadon $\left(\mathrm{D}_{3} \mathrm{zd}\right)$, Famennien inférieur, est constitué de calcaires marneux et de calcaires dolomitiques avec par endroits des intercalations d'évaporites. Son épaisseur est de $90 \mathrm{~m}$;

- Eliets $\left(\mathrm{D}_{3} \mathrm{el}\right)$, Famennien inférieur, se compose de calcaires et de calcaires dolomitiques d'une épaisseur de $180 \mathrm{~m}$. À l'est, il est essentiellement volcanogène ;

- Pétrikov $\left(\mathrm{D}_{3} \mathrm{ptr}\right)$, Famennien inférieur, est constitué d'une succession de calcaires marneux et de marnes dolomitiques de $40 \mathrm{~m}$ d'épaisseur.

Les pièges de pétrole du complexe intersalifère sont répartis dans les horizons Zadon et Eliets (Garetsky et al., 1984).
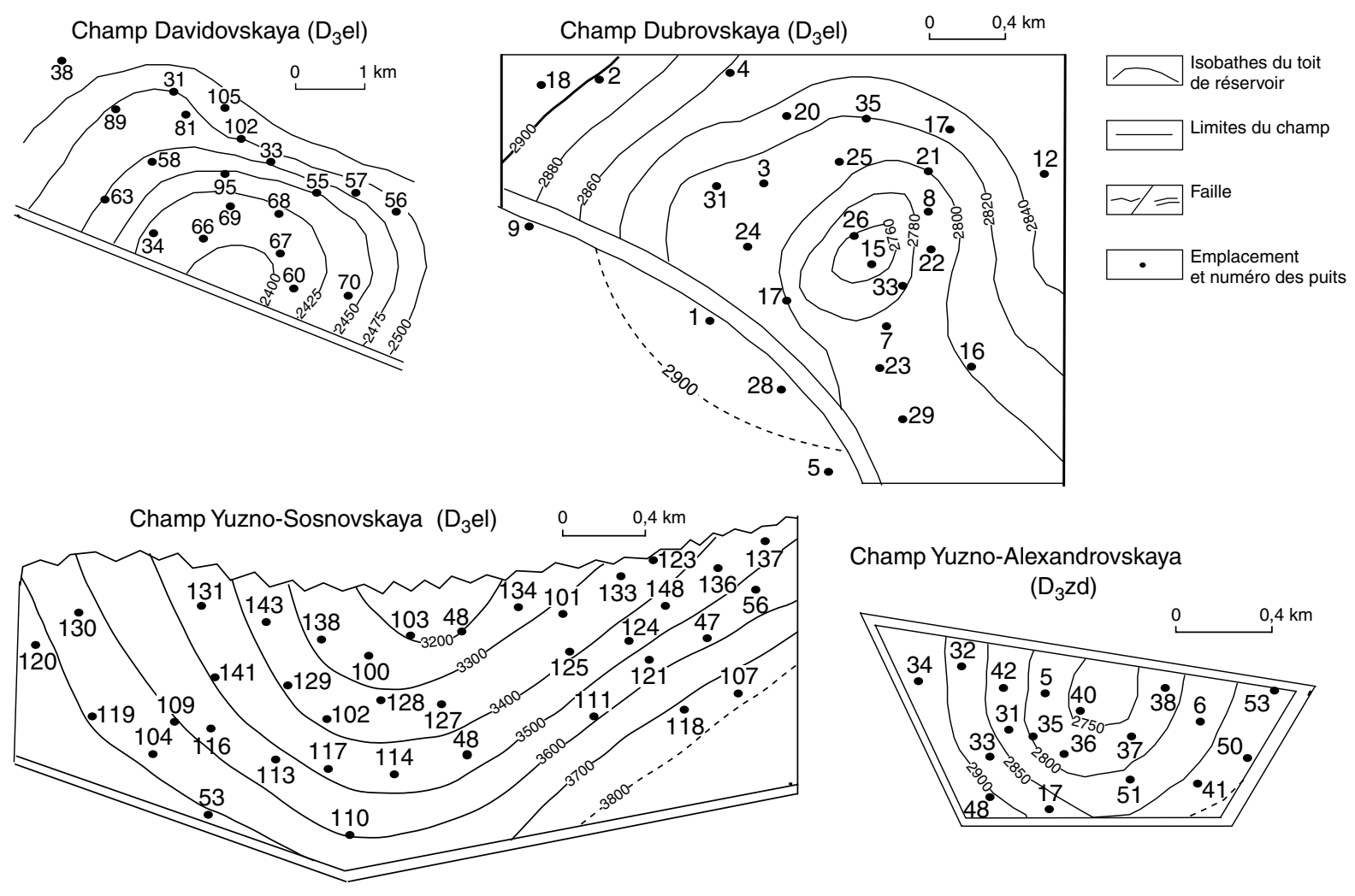

Champ Yuzno-Alexandrovskaya

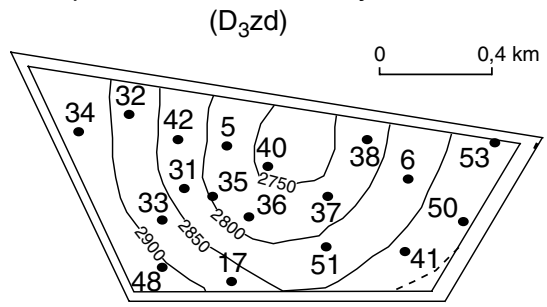

Figure 3

Cartes structurales des toits des horizons productifs intersalifères.

Structural map of oil intersalt horizons. 

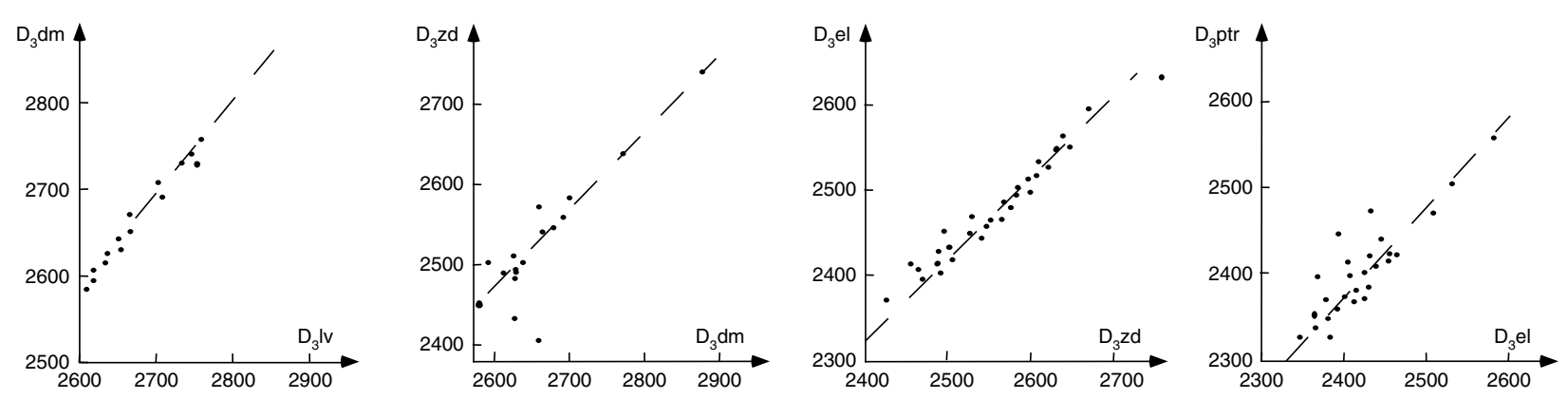

\section{Champ Yuzno-Alexandrovskaya}
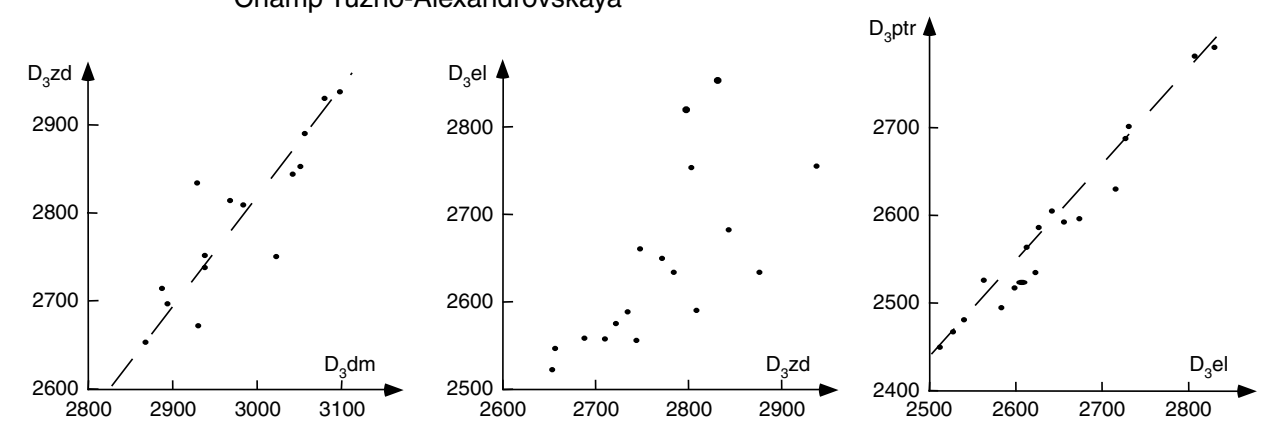

\section{Champ Dubrovskaya}
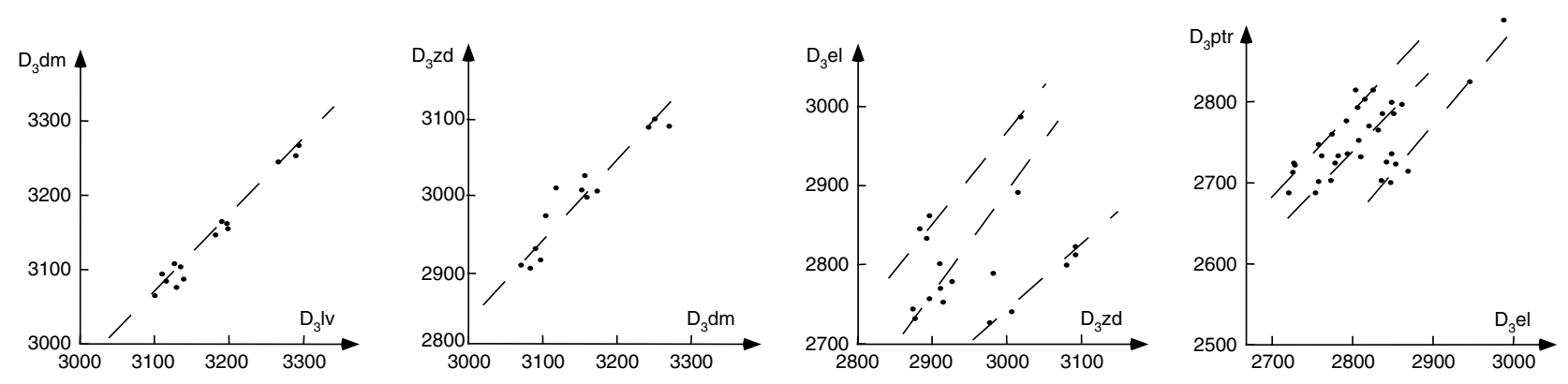

Champ Yuzno-Sosnovskaya
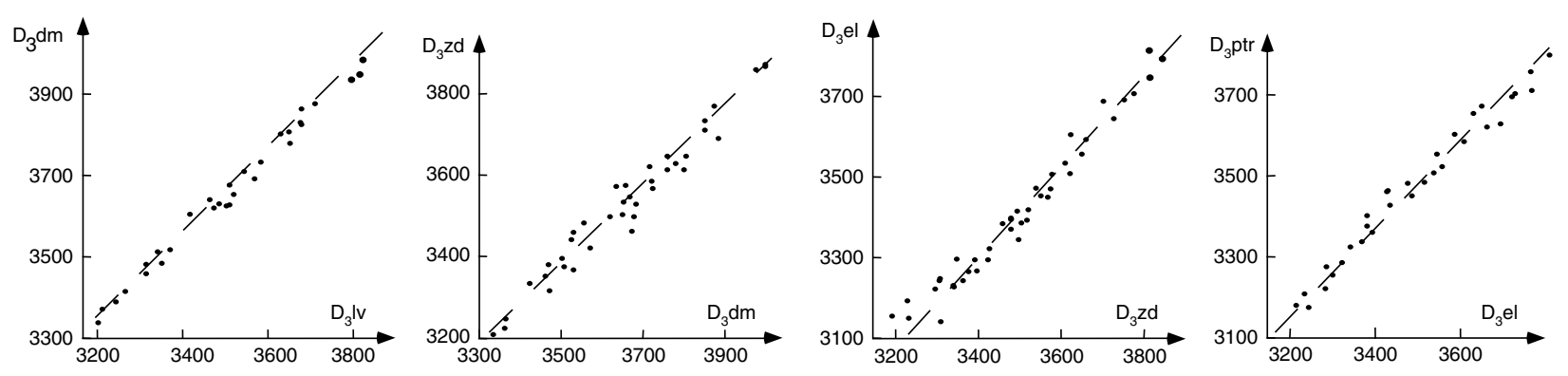

Figure 4

Graphiques de corrélation des toits des horizons intersalifères.

Correlation graphics of the intersalt horizons. 
Pour l'analyse de l'influence des processus de sédimentation et de post-sédimentation sur le plan structural, on a utilisé la méthode de corrélation des profondeurs absolues des limites stratigraphiques des horizons du complexe intersalifère. Elle est basée sur la coïncidence linéaire des profondeurs absolues des limites stratigraphiques traversées par les différents forages.

Cette méthode, peu utilisée par les géologues des pays occidentaux, permet de rendre compte, de façon synthétique, des variations d'épaisseur d'une couche en fonction de la position structurale. Ainsi, une couche d'égale épaisseur sur toute la structure se traduira par un ensemble de points alignés sur une droite de pente $l$. Pour des couches à épaisseur variable, on observe une disposition désordonnée des points, qui témoigne de l'évolution géologique des antiformes (Hafisov, 1972 ; Nesterov, 1981). La méthode permet de déterminer les blocs à différentes vitesses de sédimentation et aux constructions biogènes sur les flancs et au cœur des antiformes des réservoirs carbonatés intersalifères.

Les territoires étudiés présentent partout une bonne continuité des couches, déduite des graphiques de corrélation dans les horizons Domanovitch $\left(\mathrm{D}_{3} \mathrm{dm}\right)$ et, dans des cas particuliers, Zadon $\left(\mathrm{D}_{3} \mathrm{zd}\right)$, Eliets $\left(\mathrm{D}_{3} \mathrm{el}\right)$ et Pétrikov $\left(\mathrm{D}_{3} \mathrm{ptr}\right)$. Les disjonctions et les constructions biogènes déforment la liaison linéaire (fig. 4).

Le champ Yuzno-Alexandrovskaya présente une anomalie à aspect récifal au cœur de l'anticlinal dans les couches du Zadon (fig. 4). Le coefficient de corrélation est de 0,5, pour une épaisseur moyenne de $184,5 \mathrm{~m}$.

Sur le champ Dubrovskaya, dans les couches de l'Eliets, il se dégage une anomalie de genre d'atoll. L'horizon a une épaisseur moyenne de $176 \mathrm{~m}$ et le coefficient de corrélation entre son mur et son toit est de 0,43.

Le champ Yuzno-Sosnovskaya, bien que présentant une corrélation linéaire dans les couches du Zadon, comporte, dans cette formation, une petite construction organogène en forme d'arc dans sa partie périclinale sud. Le coefficient de corrélation est de 0,60 avec 76 m d'épaisseur moyenne de la couche.

\section{ANALYSES PÉTROGRAPHIQUES ET PÉTROPHYSIQUES}

\subsection{Description micrographique des échantillons}

L'observation et la description de 154 lames minces réalisées sur l'ensemble des horizons ont permis de regrouper les roches carbonatées en différents groupes.

\subsubsection{Calcaires bioclastiques noduleux}

Champ Davidovskaya : puits 17 (2629-2634 m/ D 3 el) ; puits $7\left(2779,9-2795,6 \mathrm{~m} / \mathrm{D}_{3} \mathrm{el}\right)$.
Champ Dubrovskaya : puits 5 (3054-3062 m/ D $\mathrm{el}$; 3073-3078 et 3087-3104 m / D $3 \mathrm{zd})$; puits 18 (3072-3080 m / $\left.\mathrm{D}_{3} \mathrm{el}\right)$.

Sur les lames, on observe des nodules et granules calcaires donnant des granulométries moyennes et grossières (de 0,2$0,5 \mathrm{~mm}$ à $1 \mathrm{~mm}$, parfois jusqu'à $5 \mathrm{~mm}$ ), de forme ronde et ovale, des plaques d'échinodermes et des radioles d'oursin, des fragments de coquilles de brachiopodes et autres bivalves, des fragments d'ostracodes et autres débris biogènes indéterminés. Les concrétions sont microcalcitiques et la paroi externe des nodules est entourée d'incrustations calcaires. Le ciment est fin calcitique et comporte souvent des témoins de structure microalgaire. Sur ces lames, on observe également des stylolites avec, par endroits, une coloration marron liée probablement à la présence des bitumes et des cavernes et fissures remplies de cristaux d'anhydrite. Parfois, dans la roche, on note la présence de petits cristaux de quartz.

\subsubsection{Calcaires fins cristallins légèrement dolomitisés, poreux et fissurés}

Champ Davidovskaya : puits 16 (2635-2667 m / D zd) ; puits 17 (2726-2731 m / D 3 zd).

Champ Dubrovskaya : puits 5 (3054-3062 m/ D 3 el ; 3087-3104 m / D $\mathrm{zd}$ ) ; puits 7 (3073-3078 m / D 3 el) ; puits 18 (3073-3078 m et 3190-3199 m / D el) ; puits 24 (3041$\left.3046 \mathrm{~m} / \mathrm{D}_{3} \mathrm{el}\right)$.

Ils sont formés d'une masse de fins cristaux de calcite. Dans la masse cristalline, on distingue nettement quelques rhomboèdres subautomorphes et automorphes de dolomite de 0,03 à $0,2 \mathrm{~mm}$ d'arête. L'analyse diffractométrique de certains échantillons a montré que ces roches contiennent 70 à $92 \%$ de calcite et 8 à $30 \%$ de dolomite.

\subsubsection{Calcaires bioclastiques oncolitiques (à algues) fortement dolomitisés et dolomies calcaires}

Champ Davidovskaya : puits 16 (2655 m / D 3 el) ; puits 17 (2731-2743 m / D zd).

Champ Yuzno-Sosnovskaya : puits 44 (3594,9-3601 m / $\mathrm{D}_{3} \mathrm{el}$; 3683-3691 m/ $\left.\mathrm{D}_{3} \mathrm{zd}\right)$; puits 48 (3680-3693 m et 3722$\left.3762 \mathrm{~m} / \mathrm{D}_{3} \mathrm{zd}\right)$; puits $56\left(3837-3863 \mathrm{~m} / \mathrm{D}_{3} \mathrm{zd}\right)$.

En lames minces, ils sont composés de divers débris, parmi lesquels des échinodermes, des bivalves et autres fragments organiques. Les oncolites ont un diamètre atteignant 6-8 $\mathrm{mm}$ et leur paroi externe est entourée de recristallisations de calcite incrustée. Les cristaux de dolomite, sous forme de rhomboèdres bien automorphes de 0,02-0,03 à 0,08-0,1 mm d'arête, se développent à travers les cristaux fins de calcite dans l'espace intergranulaire et constituent plus de $40 \%$ du volume total de la roche. L'analyse diffractométrique de quelques échantillons montre que les roches sont constituées de $42 \%$ de calcite et $58 \%$ de dolomite. 


\subsubsection{Roches anhydritiques et carbonatées à anhydrite}

Champ Davidovskaya : puits 1 (2571-2580 m et 2611$\left.2617 \mathrm{~m} / \mathrm{D}_{3} \mathrm{lv}\right)$; puits $17\left(2487-2494 \mathrm{~m} / \mathrm{D}_{3} \mathrm{el}\right)$.

Ces roches sont faites d'une masse de cristaux d'anhydrite fins prismatiques, souvent fibreux ou de forme conique. Un clivage orthogonal est caractéristique pour les cristaux allongés. Le carbonate est de nature dolomitique (rhomboèdres automorphes). De telles roches sont beaucoup plus répandues dans les horizons supérieurs $\left(\mathrm{D}_{3} \mathrm{ptr}\right)$ où elles forment des couches plus épaisses. On les retrouve dans les réservoirs sous forme de lamines de quelques centimètres d'épaisseur.

\subsubsection{Dolomies cristallines, poreuses, caverneuses et fissurées}

Champ Yuzno-Sosnovskaya : puits 44 (3521-3534 m / $\mathrm{D}_{3} \mathrm{el}$; 3604-3611 m et 3631-3645,9 m / $\left.\mathrm{D}_{3} \mathrm{zd}\right)$; puits 48 (3608$3618 \mathrm{~m}$ et 3639,5-3646,8 m/ $\left.\mathrm{D}_{3} \mathrm{el}\right)$; puits $50(4320-4339 \mathrm{~m} /$ $\mathrm{D}_{3} \mathrm{el}$ ) ; puits 53 (3951-3964 $\mathrm{m}$ et 3976-3979 $\mathrm{m} / \mathrm{D}_{3} \mathrm{el}$; 4010,7-4022,7 m / D $\mathrm{zd})$; puits $56(3736-3752 \mathrm{~m}, 3763-$ 3775,7 m, 3780,7-3789,7 m, 3790-3795,8 m, 38043809,8 m/ D el ; 3820-3824 m et 3837-3842 m / $\mathrm{D}_{3} \mathrm{zd}$ ).

Champ Yuzno-Alexandrovskaya : puits $34(2972,1 \mathrm{~m} /$ $\mathrm{D}_{3} \mathrm{el}$; 3000-3008 m / $\left.\mathrm{D}_{3} \mathrm{zd}\right)$; puits 36 (2993-3015 m / $\left.\mathrm{D}_{3} \mathrm{zd}\right)$.

Sur les lames, on observe de belles plages de cristaux fins, moyens ou grossiers, automorphes, de dolomite avec des fissures et cavernes. Dans certaines variétés, le volume des vides atteint $15-20 \%$. Sur certaines lames, on observe une masse cryptocristalline de calcite et de la matière organique en dispersion. On note la présence de quelques rares grains de pyrite et parfois de quartz. Dans certaines dolomies apparaissent des roches effusives et des feldspaths présentant des macles polysynthétiques. Les analyses diffractométriques révèlent que ces échantillons sont constitués de plus de $95 \%$ de dolomite.

\subsubsection{Roches tufogéniques à grains moyens et grossiers}

Champ Yuzno-Alexandrovskaya : puits 34 (2970,5 m, 1971,1 m, 2973,5 m, 2974,4 m et 2974,7 m / D 3 el ; 3005 m / $\left.\mathrm{D}_{3} \mathrm{zd}\right)$; puits 36 (2993-3015 m/ $\left.\mathrm{D}_{3} \mathrm{zd}\right)$.

Les roches de ce groupe, retrouvées à l'est du bassin (champ Yuzno-Alexandrovskaya), sont très poreuses et caverneuses. Les vides ont un diamètre de 0,1 à $2 \mathrm{~mm}$ et les tufs sont par endroits carbonatés. Les carbonates sont constitués de cristaux automorphes de dolomite dans une proportion atteignant parfois $40 \%$. Le matériel tufogénique est constitué de fragments de plagioclases, de feldspaths potassiques et de verre volcanique chloritisé. L'abondance des fragments chloritisés et l'absence presque absolue du quartz indiquent la nature andésito-basaltique du volcanisme. Plusieurs fragments pyroclastiques ont une coloration rougeâtre due à de l'oxyde de fer. Par endroits, sur la lame, on observe des fissures d'ouverture 0,1 à $0,3 \mathrm{~mm}$, remplies d'une masse argileuse, et des cristaux authigènes de pyrite.

En conclusion, le complexe intersalifère du bassin de Pripiat est constitué de calcaires bioclastiques contenant des serpules et nodules, de calcaires cristallins avec un faible pourcentage de débris organiques, de dolomies à structure organique et granulaire et de roches tufogéniques et carbonatées tufogéniques à l'est du bassin. La disposition désordonnée de ces diverses roches sur la verticale et la grande variation de la taille des cristaux (de cryptocristalline à grossière) témoignent de la contemporanéité de leur dépôt et des recristallisations catagénétiques. Les cavernes sont issues de la dissolution et de la recristallisation des carbonates primaires.

Les minéraux authigènes observés (quartz, pyrite, feldspaths et carbonates secondaires) se sont formés lors de la catagenèse, et ont participé à la diversification des carbonates et surtout à la variation du volume des vides dans leur masse. La répartition spatiale des échantillons décrits montre que la dolomitisation s'intensifie localement de la base vers le sommet des anticlinaux, où il $\mathrm{y}$ a plus de fissurations, et régionalement, des champs ouest vers les champs est, sous l'influence croissante du volcanisme.

\subsection{La microscopie électronique}

Le microscope électronique à balayage permet, au fort grossissement, d'étudier la forme et les dimensions des particules et roches et d'établir la présence des processus secondaires de dissolution et de recristallisation. Il offre la possibilité d'étudier génétiquement les formes acquises par la calcite, la pyrite et autres minéraux.

La série de photographies présentée en figure 5 montre l'évolution graduelle des changements observés au sein des roches carbonatées du Zadon-Eliets des réservoirs étudiés. On part des roches primaires compactes (fig. $5 a$ ) pour aboutir aux roches fragmentées, meubles et poreuses (fig. $5 b$ ). Dans les cavernes de ces dernières, il se forme de nouveaux cristaux frais de dolomite ( $f$ ig. $5 c$ et $5 d$ ). On observe également l'occupation des vides par le pétrole (fig. $5 d$ ).

Les roches carbonatées primaires sont formées de variétés homogènes assez compactes. La formation des dolomies s'est effectuée à partir des calcaires magnésiens. Selon Kazenkina (1975), en dehors du carbonate de calcium, il s'est déposé également dans le bassin, par suite d'une sédimentation mécanique et physico-chimique, divers débris détritiques et des cristaux et composés colloïdaux de fer, de silicium, de phosphore de magnésium et autres éléments issus des solutions chimiques et organiques qui ont servi à la formation de minéraux authigènes diagénétiques. La vie, dans ces sédiments, d'une masse colossale de bactéries fut un facteur biochimique très important dans la décomposition de la matière organique. Les produits de cette décomposition, 

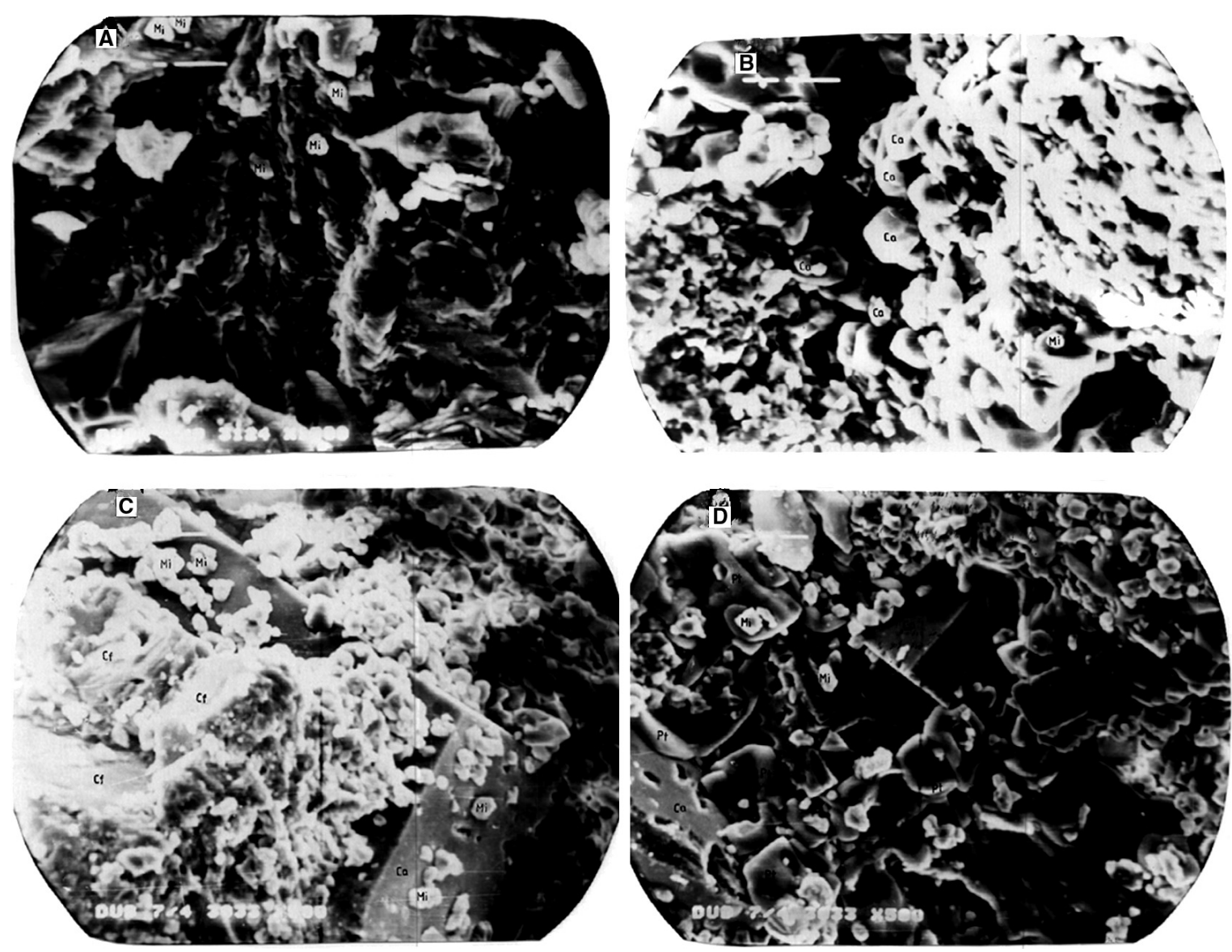

Figure 5

Planches photographiques des roches carbonatées observées au microscope électronique.

(a) $(\times 400)$ Carbonate (calcaire) primaire fortement corrodé. On observe des petits fragments de calcite (micrites) « Mi» sous forme de taches blanchâtres dans la partie supérieure du calcaire primaire en destruction.

(b) ( $\times 400)$ Roche micritique (calcaire) poreuse fissurée. Le long des fissures et cavernes « $\mathrm{Cv}$ » (zones sombres), dans les conditions de libre croissance, la micrite s'est recristallisée, formant de nouveaux cristaux de calcite « $\mathrm{Ca} »$ bien automorphes (limites des faces bien nettes) de 10 à $15 \mathrm{~mm}$ de diamètre.

(c) $(\times 200)$ Calcaire microgrenu avec inclusion de gros cristaux de calcite. On observe à gauche les résidus de la dissolution complète d'une calcite feuilletée « $\mathrm{Cf}$ », ayant conduit à la formation d'une masse microlitique « $\mathrm{Mi}$ » (2-3 mm). Dans cette dernière, est enfoui un gros cristal de calcite secondaire « $\mathrm{Ca}$ » de $160 \mathrm{~mm}$ d'arête. Bien qu'automorphe, ce cristal présente déjà des traces de dissolution future (faces corrodées).

(d) $(\times 200)$ Cristaux de calcite fortement corrodés et dissous donnant la micrite « Mi ». On observe en haut et en bas à gauche de nouveaux cristaux de calcite « $\mathrm{Ca}$ » à arêtes et faces corrodées et des gouttes de pétrole « $\mathrm{Pt}$ » entourant parfois des grains de calcite sans les imbiber : ceci pourrait témoigner du caractère hydrophile de la roche magasin, mais la préparation des échantillons peut fortement perturber la mouillabilité.

Photomicrographs of carbonate rocks.

(a) (× 400) Highly corroded primary carbonate (limestone). Small fragments of calcite (micrites) "Mi" can be seen in the form of withish spots in the upper part of the primary limestone that is being broken down.

(b) $(\times 400)$ Fractured porous micritic (limestone) rock. Along the fractures and cavities " $C v$ " (dark areas), under free-growing conditions, the micrite has recrystallized forming new calcite crystals "Ca". They are automorphic (the edges of their faces are distinct) and have a diameter of 10 to $15 \mathrm{~mm}$.

(c) $(\times 200)$ Microgranular limestone with inclusions of large calcite crystals. On the left are residues of the complete dissolution of a foliated calcite " $C f$ " that led to the formation of a microlitic mass "Mi" (2-3 mm). A large (edge $160 \mathrm{~mm}$ long) crystal of secondary calcite "Ca" is enclosed in this mass. Although the crystal is automorphic, it already shows traces of future dissolution (corroded faces).

(d) $(\times 200)$ Highly corroded and dissolved calcite crystals giving micrite "Mi". At the top and bottom left, new calcite crystals "Ca" with corroded edges and faces can be seen. There are also drops of petroleum "Pt" that sometimes surround the calcite grains without imbibing them. This might evidence the hydrophilic nature of the reservoir rock, but the preparation of macroscopic samples can greatly distort wettability. 
surtout le $\mathrm{CO}_{2}$, le $\mathrm{CH}_{4}$ et le $\mathrm{NH}_{3}$, ont contribué à la diminution du $\mathrm{pH}$ de la solution vaseuse et à la dissolution d'une partie des carbonates sédimentés, qui sont passés ainsi à l'état de bicarbonates. Aux étapes suivantes de la diagenèse, par suite d'une arrivée de $\mathrm{CO}_{2}$ supplémentaire dans la solution, les carbonates reprécipitent sous forme calcitique ou dolomitique. Mentionnons qu'à l'est du bassin où se situe le champ Yuzno-Alexandrovskaya, les couches du Zadon sont comprises entre les formations volcaniques des horizons antérieur (Domanovitch) et postérieur (Eliets). Ainsi la formation des dolomies dans cette partie du territoire s'est-elle effectuée dans des conditions de haute pression,en présence d'acide carbonique ayant favorisé l'ordonnancement structural des roches calcaromagnésiennes.

À la catagenèse, sous l'effet de la température et sous l'action des eaux souterraines, le massif carbonaté est soumis à diverses transformations : la dissolution et la reprécipitation des carbonates et d'autres minéraux, la micritisation et la dolomitisation de la calcite. Ces transformations s'observent aussi bien sur les calcaires primaires et secondaires que sur les dolomies secondaires.

\subsection{Méthodes antérieures d'interprétation des données diagraphiques}

Dans le souci de maîtriser les particularités des réservoirs pour une meilleure productivité, plusieurs méthodes géophysiques de diagraphie (électrique, acoustique, gamma, neutron-gamma et cavernométrie) ont été utilisées dans l'intervalle du complexe carbonaté intersalifère. Les diagrammes obtenus ont été soumis à diverses interprétations en comparaison avec les données de carottage. C'est ainsi que le couplage des méthodes acoustiques $(\log \Delta T)$ et neutron-gamma (intensité $n \gamma$ ) permet la subdivision lithologique du massif carbonaté en intervalles calcaires, dolomitiques évaporitiques ou intermédiaires, avec des pourcentages correspondants (Anpilogov, Gossoudareva et al., 1981 ; Zaliaev et al., 1981).

Les résultats les plus précieux ont trait à la porosité et à la teneur en argile déterminées par le traitement couplé des données normées des méthodes acoustiques, gamma et neutron-gamma. Ainsi, il est montré que la porosité des calcaires varie entre 1,5 et $7 \%$ avec une moyenne de $4 \%$ et celle des dolomies de 3 à $13 \%$ avec une moyenne de 6,5\% . Quant à la porosité ouverte, elle varie de 0,5 à $5 \%$ (avec une moyenne de $1,5 \%$ ) pour les calcaires. Pour les dolomies, elle varie de 1 à $7,5 \%$ mais peut atteindre toutefois $11,5 \%$ et parfois $20 \%$ dans les zones vacuolaires (Anpilogov et al., 1987 ; Zaliaev et al., 1981).

L'analyse qualitative des types de porosité est réalisée à partir de l'interprétation des diagrammes des méthodes électriques, acoustiques et neutron-gamma comparés aux données des carottes. Ainsi, pour la porosité diagraphique à dominance intergranulaire, les valeurs présentent une coïncidence quelle que soit la méthode utilisée, alors que les réservoirs vacuolaires sont caractérisés par des valeurs plus élevées de la porosité neutron par rapport aux autres méthodes. Les fissures influent sur les valeurs de la résistivité électrique $(\rho)$ et de la différence de temps acoustique $(\Delta T)$ des roches qui les contiennent.

La teneur en argile dans les carbonates induit une augmentation de l'intensité gamma et une diminution de la porosité (Anpilogov et Gossoudareva, 1975). Les courbes cumulatives de porosité et de teneur en argile donnent par leur intersection la valeur de la porosité limite entre les roches magasins et les couvertures. Celle-ci varie entre 2,5 et $7,5 \%$, soit en moyenne 4,8\% correspondant à une teneur limite en argile de $15 \%$ (Anpilogov et Gossoudareva, 1987; Mossiouk et Araktchev, 1976).

S'il est vrai que la porosité diminue avec l'augmentation de la teneur en argile, la subdivision des couches en roches magasins et roches couvertures à partir de la porosité et de la teneur en argile ne tient pas compte de la perméabilité et de son évolution avec la baisse de la pression des couches. C'est d'ailleurs ce qui explique la non-concordance entre les réservoirs dégagés et les résultats d'essai de débit. La porosité ouverte, qui varie avec la nature et l'ampleur des transformations secondaires au sein des carbonates, devra servir de base pour la subdivision des roches magasins et couvertures.

\section{DENSIMÉTRIE DYNAMIQUE DES ROCHES CARBONATÉES (PRISE D'EAU PAR IMBIBITION SPONTANÉE) ET TYPISATION DES RÉSERVOIRS NATURELS}

L'étude de la densimétrie dynamique est inspirée de la méthode de Starastin (Divakov et al., 1984), qui consiste en la saturation des échantillons de roche à l'eau distillée, leur pesée périodique sur une balance technique jusqu'à saturation totale, puis leur étude ultrasonore. Ainsi, à partir des paramètres physico-mécaniques des roches et des vitesses de propagation des ondes ultrasonores, on détermine leur porosité efficace et autres caractéristiques physicomécaniques.

Cette méthode a été améliorée au laboratoire de géologie appliquée du Département des minéraux utiles et de leur exploration (Université russe de l'amitié des peuples) par Divakov et al. (1984), pour une détermination plus facile de la porosité. À la place d'une balance technique pour la lecture de la courbe de saturation, on utilise un densimètre permettant d'effectuer des mesures plus rapides, d'éviter quelques erreurs et d'obtenir une nouvelle information qualitative sur le caractère de saturation des pores et fissures 
(Divakov et al., 1984). Sur l'appareil, on lit directement la densité de l'échantillon, et sa porosité à tout instant $\imath$ de la saturation se calcule selon la formule :

$$
K_{\mathrm{\imath}}=\frac{\sigma_{i}-\sigma_{0}}{\sigma_{0}}
$$

avec :

$\sigma_{i} \quad$ densité après un temps $i$ de saturation, en $\mathrm{kg} / \mathrm{m}^{3}$

$\sigma_{0} \quad$ densité initiale de l'échantillon à sec, en $\mathrm{kg} / \mathrm{m}^{3}$

$K_{1} \quad$ porosité ouverte de l'échantillon à chaque instant $\mathrm{l}$

\subsection{Principe de la densimétrie dynamique (prise d'eau par imbibition spontanée)}

L'échantillon à étudier est auparavant séché dans une étuve pendant deux jours à la température de $105^{\circ} \mathrm{C}$, en vue d'en enlever les eaux libres et pelliculaires. Cependant, restent conservées dans l'échantillon, l'eau hygroscopique dont la température de destruction est supérieure à $250^{\circ} \mathrm{C}$ et, en partie, l'eau pelliculaire ayant conservé une liaison structurale avec l'eau hygroscopique. Ainsi la saturation en air est-elle voisine de la porosité ouverte.

Les mesures de la densité sont effectuées immédiatement après émersion de l'échantillon dans l'eau, puis répétées après 15,30 minutes ; 1, 2, 4, 6 heures ; et ensuite 1, 2, 3, etc., jours, jusqu'à saturation totale, marquée par la stabilisation de la densité.

Par calcul, les densités sont converties en porosités selon la formule mentionnée ci-dessus et, pour chaque échantillon, on construit le graphique de saturation dans le temps. Les graphiques ont une configuration différente, l'élément commun étant la stabilisation de la saturation, à partir de laquelle on détermine la porosité ouverte de l'échantillon. Le comportement des graphiques sur des courtes durées de saturation est caractérisé par une augmentation rapide de la masse volumique, due au remplissage des pores. Les premières minutes correspondent au remplissage des plus grands pores, canaux et fissures. La vitesse de saturation est déterminée par l'angle initial que forme le graphique avec l'axe des abscisses.

Pour le présent travail, 89 échantillons de roches carbonatées du massif intersalifère du Dévonien supérieur ont été étudiés. La saturation des échantillons a été complète à partir du $6^{\mathrm{e}}$ jour pour certains et a duré jusqu'au $35^{\mathrm{e}}$ jour pour d'autres. Pour une analyse plus commode, les données obtenues ont été classées par affinité d'allure des courbes et des valeurs maximales de porosité à l'ordinateur selon le programme Claster Analyse (Duran et Odell, 1977). Ceci nous a permis de répartir nos courbes en sept classes contenant chacune 2 à 53 échantillons (fig. 6). Il a été calculé pour chaque classe la valeur moyenne de leur porosité à tous les instants $\imath$ de leur saturation, ainsi que le coefficient de porosité ouverte (kpo).

\subsection{Analyse des résultats de groupage}

La classe 1 comprend 10 échantillons, dont 4 calcaires dolomitisés et 6 dolomies cristallines à grains fins et moyens. Ils ont un kpo de $4 \%$ et forment un réservoir complexe poreux, vacuolaire et fissuré. Le graphique montre une saturation régulière jusqu'au $8^{\mathrm{e}}$ jour, où intervient la saturation totale. Les vides dans ces échantillons seraient uniformément répartis, ce qui explique leur saturation régulière et rapide (fig. 6).

La classe 2 comprend 2 échantillons de dolomies calcaires à grains moyens, poreux, caverneux et fissurés. Leur porosité ouverte est de $4,8 \%$. Ils sont caractérisés par une saturation rapide et régulière jusqu'au $2^{\mathrm{e}}$ jour, suivie d'une saturation lente du $2^{\mathrm{e}}$ au $20^{\mathrm{e}}$ jour (fig. 6). Le premier intervalle correspondrait au remplissage des vacuoles et grands pores et le second au remplissage de l'espace intergranulaire et des fissures.

Dans la classe 3 sont classés 2 échantillons de calcaires bioclastiques, oncolitiques, argileux, noduleux, à serpules et fissurés. Ils sont caractérisés par un kpo de 3,7\%, une saturation rapide et complète des premières minutes à la $4^{\mathrm{e}}$ heure (fig. 6). Ici, le rôle important dans la saturation serait, selon toute observation, lié aux fissures et aux rares cavernes de dissolution des organismes.

La classe 4 est représentée par un seul échantillon de roche dolomito-anhydritique qui, au cours de la saturation, s'est décomposé en plaquettes séparées. L'échantillon s'est saturé brusquement à la première heure, puis régulièrement

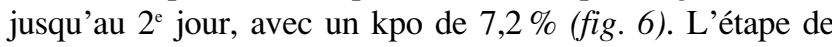
saturation brusque correspond à celle de la partie anhydritique et l'étape de saturation régulière à la saturation de la partie dolomitique de la roche.

Dans la classe 5 entrent 4 échantillons de dolomies cristallines poreuses, vacuolaires et bitumineuses. Leur kpo est de $10,6 \%$. La valeur élevée du kpo témoigne du caractère très poreux des roches. La saturation est très lente le premier jour, puis assez rapide pendant 25 jours et plus (fig. 6). Certainement, la saturation très lente des premières 24 heures serait liée au fait que les échantillons séchés contiendraient encore des huiles qui auraient rendu difficile au départ le mouvement de l'eau distillée froide dans l'échantillon.

Il faut noter que l'imbibition spontanée est fortement dépendante de l'état de mouillabilité à l'eau des échantillons séchés. Cette mouillabilité étant dépendante des conditions de nettoyage des échantillons, ceux-ci, constitués de fins cristaux, pourraient avoir une surface moins favorable à la rapide imbibition les premières heures.

Dans la classe 6, figurent 17 échantillons de dolomies (14) et de calcaires (3) fins cristallins poreux et bitumineux, avec un kpo de 4,7\%. Le graphique de saturation croît lentement avec une légère accélération après les premières 24 heures et la saturation complète intervient au bout de 30 jours (fig. 6). Les échantillons de la $6^{\mathrm{e}}$ classe seraient de même nature que 

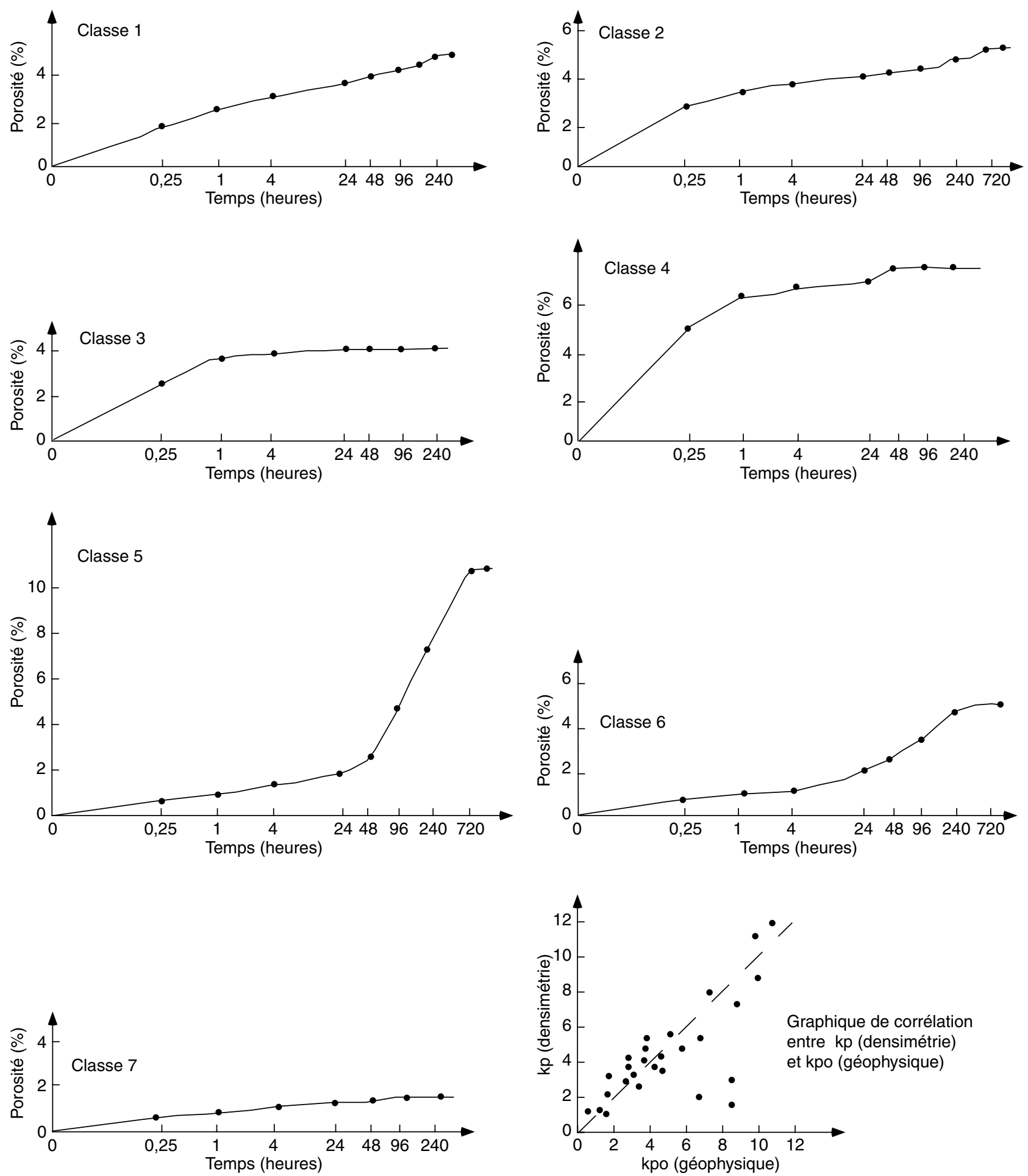

Figure 6

Courbes de saturation en eau des roches carbonatées.

Saturation graphics of carbonate rocks. 
ceux de la $5^{\mathrm{e}}$, à la différence que ceux-ci sont moins poreux à cause de leur plus grande compaction, liée au diamètre plus petit de leurs grains.

La classe 7 regroupe 53 échantillons de calcaires argileux. Ils ont un kpo de $1,7 \%$ et présentent une saturation régulière pendant 15 jours (fig. 6). Les roches de cette classe appartiennent aux variétés argileuses denses constituant les fausses couvertures et les écrans.

L'analyse comparée des porosités issues de la saturation dynamique des échantillons et de celles issues de
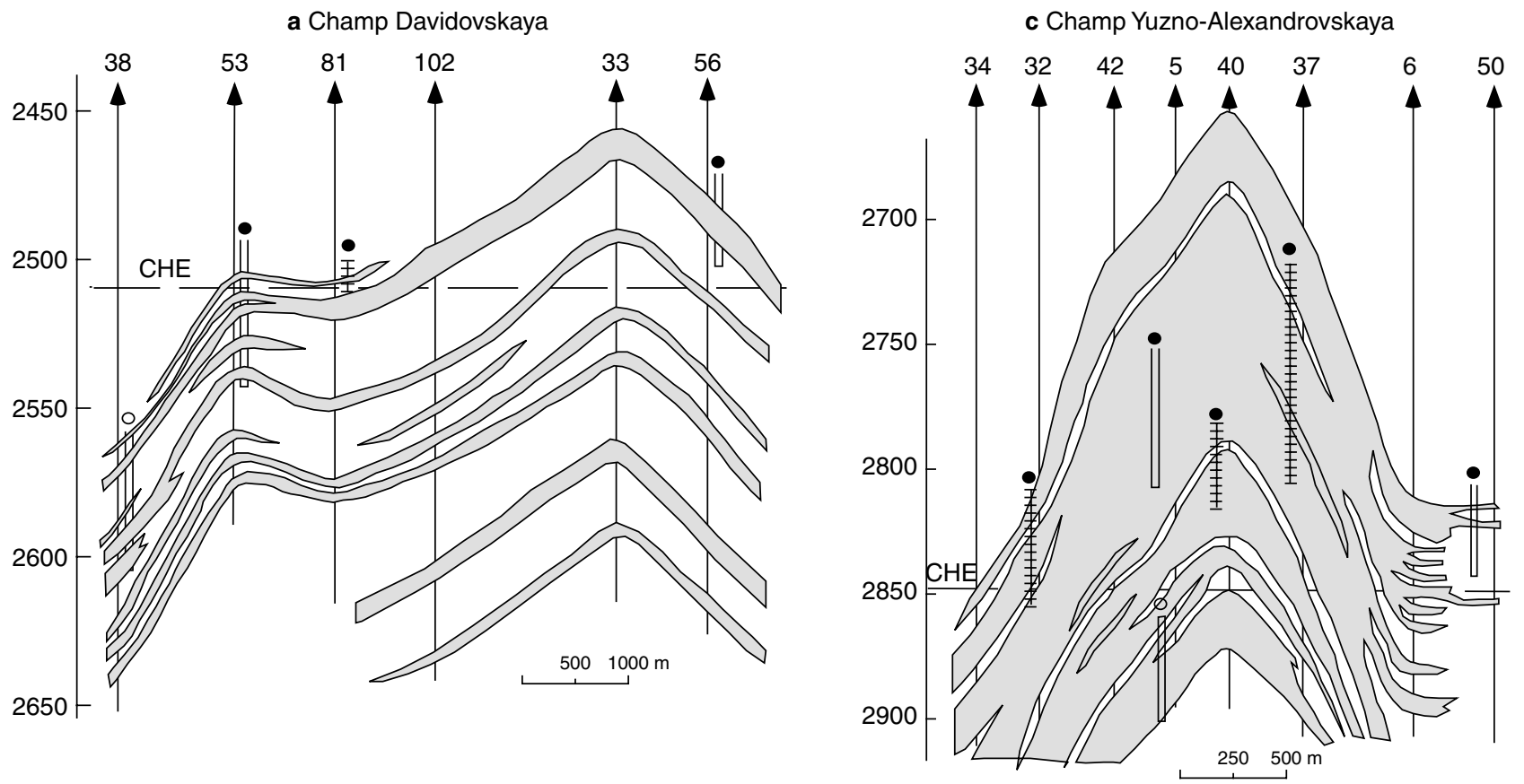

b Champ Dubrovskaya
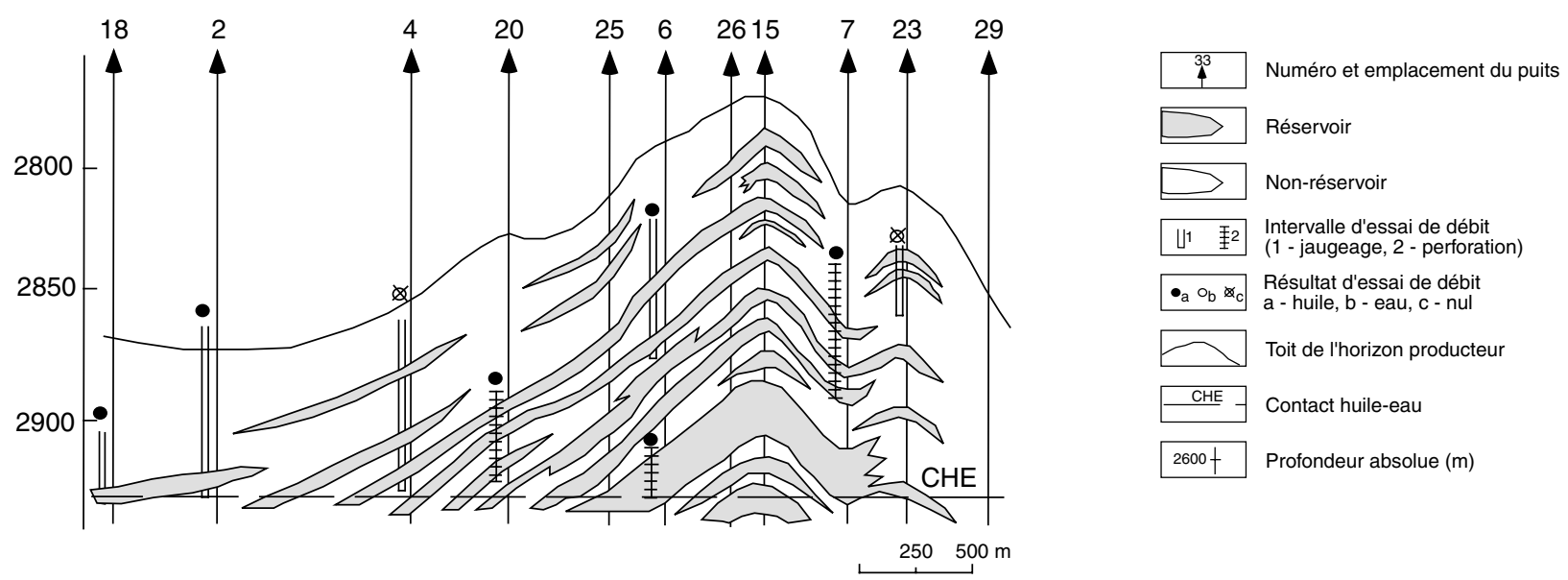

Figure 7

Coupes schématiques des différents types de réservoirs naturels carbonatés.
(a) Type tabulaire homogène.
(b) Type tabulaire hétérogène.
(c) Type massif homogène.

Schematic cross-sections of different natural reservoirs types.

(a) Tabular homogeneous.

(b) Tabular heterogeneous.

(c) Massif homogeneous. 
l'interprétation géophysique (porosités neutrons), obtenues sur le chantier, montre que la porosité déterminée par densimétrie correspond à la porosité ouverte. Le graphique de dépendance entre ces deux porosités présente une bonne corrélation (fig. 6).

En somme, les roches étudiées ont dans l'ensemble une masse volumique de 2,4 à $2,78 \mathrm{~kg} / \mathrm{m}^{3}$ pour les roches carbonatées et de $2,93 \mathrm{~kg} / \mathrm{m}^{3}$ pour les évaporites. Elles renferment des vides (pores, vacuoles et fissures) ayant une structure d'emplacement et d'interrelation qui détermine le mode de circulation des fluides en leur sein ainsi que l'allure de la courbe de saturation. La porosité ouverte varie de 0,7 à $12 \%$, avec au plus $5 \%$ pour les calcaires. Les hautes valeurs de porosité (jusqu'à $12 \%$ ) sont fixées dans les dolomies des champs pétroliers Yuzno-Sosnovskaya et de Yuzno-Alexandrovskaya. Les échantillons prélevés des parties réservoirs (essais de débit positifs) sont caractérisés par une petite valeur de la masse volumique $\left(\sigma_{0}<2,7 \mathrm{~kg} / \mathrm{m}^{3}\right)$ et une porosité supérieure à $2 \%$.

\subsection{Typisation des réservoirs naturels}

L'utilisation des données de porosité neutron issues de l'interprétation des logs diagraphiques permet de concevoir, pour la première fois en Biélorussie, des schémas détaillés des réservoirs naturels carbonatés. En effet, les données sur la porosité et la teneur en argile, dont la détermination est basée sur les méthodes Schlumberger d'interprétation diagraphique (méthodes acoustiques, gamma et neutron-gamma), servaient à déterminer la nature des roches magasins des réservoirs naturels (Anpilogov et Gossoudareva, 1987), jusque-là tous considérés comme massifs du fait de l'horizontalité du contact huile-eau.

Pour la construction des modèles de réservoirs naturels, on a procédé à la corrélation des horizons suivant des profils choisis. Ensuite, pour chaque puits, suivant que la porosité de chaque mince couche traversée était supérieure ou inférieure à $2 \%$, nous avons dégagé les intervalles des roches magasins et ceux des fausses couvertures. Enfin, la corrélation des intervalles dégagés a permis d'obtenir des schémas des réservoirs naturels qui tiennent compte de leur hétérogénéité. Selon la répartition des couches réservoirs et non-réservoirs, ces schémas ont été classés en trois types de réservoirs naturels :

- le type tabulaire homogène décelé sur le champ Davidovskaya est situé à l'est de notre zone d'étude. Les roches magasins et fausses couvertures ont des épaisseurs constantes et une bonne continuité spatiale. Elles forment verticalement sur la coupe une alternance de couches réservoirs et fausses couvertures (fig. $7 a)$;

- le type tabulaire hétérogène est localisé dans la partie centrale sur les champs Dubrovskaya et YuznoSosnovskaya. Les éléments d'hétérogénéité (roches magasins et fausses couvertures) ont une répartition très variée, tant horizontalement que verticalement. Leur succession et leur continuité sont irrégulières (fig. $7 b$ );
- le type massif homogène à l'est du bassin est repéré sur le champ Yuzno-Alexandrovskaya. Il s'agit d'un énorme massif carbonaté à aspect récifal ayant une répartition massive des roches magasins au centre de la structure antiforme. Les fausses couvertures sont abondantes en périphérie, mais elles se rencontrent rarement, et sous forme de très minces lentilles à effet insignifiant, au cœur de l'anticlinal (fig. 7c).

Au cours de l'exploitation, les gisements du premier type peuvent se transformer en un système de couches réservoirs isolées. Ici, la projection des puits pétroliers et la détermination des intervalles productifs se font assez simplement. Seulement, lors de l'exploitation des gisements du deuxième type, on assiste à leur cloisonnement, formant ainsi des couches réservoirs localisées, structurellement peu contrôlées. L'exploitation doit se faire selon un schéma individuel tenant compte de l'hétérogénéité des réservoirs naturels et des corrections permanentes de leur modèle. Pour le type massif homogène, fait d'épaisses couches de bioherme, la projection des puits pétroliers et la détermination des intervalles perforables se font selon un modèle simple et n'exigent pas de fortes corrections.

\section{CONCLUSION}

Lors de la sédimentation des horizons Zadon et Eliets du complexe intersalifère, le bassin de Pripiat était de faible profondeur, de salinité normale, avec un fond fortement disséqué sur lequel se développait une biomasse algaire.

L'abondance des carbonates à algues témoigne du fait que le facteur primordial dans la formation des roches mères est la précipitation biochimique du carbonate primaire, qui serait un mélange hétérogène de carbonate de calcium et de magnésium. Les transformations catagénétiques de ces roches ont abouti à la formation de roches magasins : dolomies et calcaires dolomitiques poreux, caverneux et fissurés. Ces transformations secondaires se sont effectuées de façon partiellement orientée, en fonction de la composition lithologique des roches primaires et de la structure des canaux de circulation des fluides, s'intensifiant localement, avec l'augmentation des fissurations, de la base vers le sommet des anticlinaux, et régionalement, de l'ouest vers l'est du bassin, sous l'influence croissante du volcanisme. La dissolution suivie de la formation des vacuoles améliore la porosité des roches carbonatées, tandis que la recristallisation et la formation des cristaux de carbonates secondaires et de sels (halite, gypse, anhydrite) tendent à la réduire, voire parfois à la supprimer.

Sur le plan porosité et perméabilité, il se dégage sept classes de roches, parmi lesquelles les calcaires microgranulaires peu poreux $(\mathrm{kpo}<2 \%)$ servent de fausses couvertures et les dolomies et calcaires dolomitiques poreux, caverneux et fissurées (kpo $>2 \%$ ), de roches magasins. Le rôle des fausses couvertures peut varier selon l'ouverture ou la fermeture des fissures qui parcourent celles-ci. La valeur $2 \%$ de la porosité représente la limite entre les 
roches magasins et les fausses couvertures, dont la répartition irrégulière détermine l'hétérogénéité des réservoirs naturels au niveau macroscopique.

Selon la disposition et la continuité des réservoirs et des fausses couvertures dans différentes localités, il se dégage trois types de réservoirs naturels : tabulaire homogène à l'ouest, tabulaire hétérogène au centre et massif homogène à l'est de la zone d'étude. La prise en considération de cette hétérogénéité fera accroître la durée d'exploitation des gisements pétroliers.

\section{REMERCIEMENTS}

Nous adressons nos plus vifs remerciements aux professeurs Postnikova et Kouznetsov pour les remarques qu'ils ont bien voulu faire sur la première forme de notre travail et les conseils précieux sur l'orientation scientifique et pratique des futurs travaux. Nous sommes reconnaissants envers le professeur Bezborodov et le maître-assistant Divakov, sous la direction desquels se sont effectuées les analyses pétrographiques et densimétriques.

\section{RÉFÉRENCES}

(Les ouvrages consultés sont pour la plupart en russe)

Abdoulikov, Y., Lahniouk, V. et Svihnouchine, N. (1978) Détermination de la teneur en argile des roches carbonatées de la dépression de Pripiat. Géologie du pétrole et gaz, 8, 54-57.

Anpilogov, A. et Gossoudareva, A. (1974) Subdivision stratigraphique des couches dévoniennes du bassin de Pripiat à partir des données géophysiques. Communication de l'Académie biélorusse des sciences, 18, 8, 744-747.

Anpilogov, A. et Gossoudareva, A. (1975) La liaison entre teneur en argile et propriétés pétrophysiques des roches carbonatées à partir du gamma-carottage. Communication de l'Académie biélorusse des sciences, 19, 11, 1038-1040.

Anpilogov, A. et Gossoudareva, A. (1987) Interprétation géologique du matériel géophysique industriel de la dépression de Pripiat, Éd. Sciences et Techniques, Minsk, 71-74.

Ayzberg, R., Garetsky, R. et Konichev, V. (1974) Perspectives pétrolières du bassin de Pripiat en fonction de sa tectonique particulière. Géologie du pétrole et gaz, 9, 8-16.

Divakov, V., Emelianov, S. et Petrov, A. (1984) Complément aux méthodes d'étude des propriétés pétrographiques des roches, Université de l'amitié des peuples, Moscou, 5-11.

Duran, B. et Odell, P. (1977) Claster Analyse, Éd. Statistique, Moscou (trad. russe de l'anglais).

Garetsky, R., Boborykine, A. et Boguino, V. (1984) Résultats essentiels des travaux d'exploration et d'exploitation pétrolière dans la dépression de Pripiat : méthodes et orientation, Éd. Sciences et Techniques, Minsk, 6-28.

Gorelik, Z. (1968) Structure actuelle et histoire de la formation tectonique du bassin de Pripiat, Éd. Sciences et Techniques, Minsk.

Haine, V. et Sokolov, B. (1991) Récifogenèse et potentialités pétrolifères : problèmes essentiels. Journal géologique, 5, 9-11.
Hafizov, F. (1972) Étude de l'histoire des structures locales par les données de l'analyse corrélationnelle des épaisseurs des couches, in Nouvelles Données sur la géologie et les potentialités pétrolifères de la Sibérie occidentale, Tumène, 247-256.

Kaki, C. (1993) Particularités de la constitution des réservoirs naturels carbonatés de pétrole dans le complexe intersalifère du bassin de Pripiat. Thèse, Université russe de l'amitié des peuples.

Karsev, I., Nazarova, N., Kazenkina, G. et Demidovitch, L. (1972) Participation des calcaires à algues aux potentialités pétrolières des formations intersalifères dévoniennes au nord-est du bassin de Pripiat. Communication de l'Académie des sciences de la Biélorussie, 16, 2, 158-161.

Kazenkina, G. (1975) Les minéraux authigènes dans les roches magasins carbonatées des horizons Zadon et Eletz comme indicateur du temps de la migration du pétrole, in Potentialités huilières de la dépression de Pripiat, Travaux scientifiques, Minsk, 104-111.

Mahnatch, A., Moskvitch, V., Kroutchek, S. et Ouriev, I. (1984) Constructions organogènes du Dévonien en Biélorussie, Éd. Sciences et Techniques, Minsk, 128-236.

Massiouk, V. et Araktchev, N. (1976) Critères de la subdivision des couches carbonatées en réservoirs et non-réservoirs à partir de la porosité et de la teneur en argile. Journal de l'Institut de recherches géologiques et pétrolières de Biélorussie, 3-21.

Mikoutsky, S., Boguino, V. et Fomkin, K. (1974) Problème des structures récifales dans le bassin de Pripiat. Problèmes de la tectonique du bassin de Pripiat, Éd. Sciences et Techniques, Minsk, 130-135.

Moskvitch, V. (1990) Régularité tectonique des formations carbonatées d'anciennes plates-formes, Éd. Sciences et Techniques, Minsk, 132-135.

Nazarova, N.V. (1975) Structure de l'espace vide des roches carbonatées, in Potentialités huilières de la dépression de Pripiat, Travaux scientifiques, Minsk, 84-99.

Nesterov, I. (1981) Régularité de la liaison entre les surfaces stratigraphiques dans la sédimentation naturelle des bassins de plateforme. Problèmes théoriques et méthodiques en géologie du pétrole et gaz, 35-43.

Ouriev, I., Mahnatch, A. et Kroutchek, S. (1974) Faciès des calcaires à algues du Zadon-Eliets du complexe carbonaté intersalifère du bassin de Pripiat. Communication de l'Académie des sciences de la Biélorussie, 18, 10, 917-920.

Postnikov, E. et Andruchenko, A. (1990) Sur les changements lithologiques et faciaux du massif carbonaté du Dévonien dans la dépression de Pripiat. Lithologie et minéraux utiles, 4, 17-23.

Pap, A. (1977) Le socle cristallin de la Biélorussie, Éd. Nèdre, Moscou.

Sahibgareev, R. et Moskvitch, V. (1975) Influence des conditions hydrodynamiques de sédimentation de la matière organique sur la formation de l'espace vide des roches magasins du Zadon dans le bassin de Pripiat. Communication de l'Académie des sciences de l'URSS, 219, 1, 201-203.

Zaliaev, N.Z., Kaoufemane, R.M. et Mamara, L.P. (1981) Interprétation complexe des paramètres géophysiques et transformations fonctionnelles informatisées. Journal de l'Institut de recherches géologiques et pétrolières de Biélorussie, 137-150.

Manuscrit définitif reçu en avril 2000 